\title{
Mathematical model of a communication channel in urban environment
}

\author{
Dmitry Chernyshov ${ }^{1, *}$, and Valentin Fedosov ${ }^{2}$ \\ ${ }^{1}$ Don State Technical University, 344000 Rostov-on-Don, Russia \\ ${ }^{2}$ Southern Federal University, 344006 Rostov-on-Don, Russia
}

\begin{abstract}
This article discusses the mechanism of signal propagation in urban environment and for the development of a three-dimensional communication channel model.
\end{abstract}

\section{Introduction}

To test the efficiency of signal processing techniques and improve their efficiency hanging can use simulation, which shows performance processing method depending on the conditions and signal propagation environment. To carry out simulation must consider the mechanism of signal propagation, for this used the communication channel model. In urban environment conditions are dominated by the multipath propagation of radio waves, as well as the phenomenon of multiple reflections, which on one hand facilitates signal transmission in the shadowing region, and on the other leads to inter-symbol interference. Thus, for the most accurate verification of the efficiency of signal processing methods in urban environment, a communication channel model is necessary in which all the above conditions are taken into account. Mainly used planar model of the communication channel, taking into account the signal propagation only in azimuth [1-4]. The purpose of this article is to analyze the signal propagation mechanism in urban areas to develop a threedimensional communication channel model.

\section{Channel model}

In general, the communication channel model can be written using expression:

$$
y(t)=\int_{-\infty}^{+\infty} h(t) \cdot x(t-\tau) d \tau+\eta=h(t) \otimes x(t)+\eta,
$$

where $h(t)$ - the impulse response of the communication channel; $x(t)$ - the transmitted signal; $y(t)$ - received signal; $\eta$ - additive noise.

\footnotetext{
* Corresponding author: dimcher@inbox.ru
} 
The spread signal in urban areas is very different from the signal propagation in free space. In $[5,6]$ it was shown that the signal is in extends essentially along the streets due to multiple reflections, as well as due to diffraction from the edges of roofs and buildings.

The general model of a channel with discrete multipath can be written using the expression:

$$
y(t)=\sum_{m=1}^{M} h_{m}(t) s\left(t, \tau_{m}\right)+\eta,
$$

where $M$ - number of signal propagation paths, $\tau_{l}$ - delay of the $m$-th path.

In this case, we can distinguish the following components of the signal propagating in urban areas: a signal propagating within the line of sight $s_{\text {los }}(t)$, a reflected $s_{\text {ref }}(t)$ and scattered signal, a signal propagated due to diffraction $s_{d i f}(t)$, and a combination thereof $\left(s_{r d}(t), s_{d d}(t), s_{r r}(t), s_{d r}(t)\right)$. An illustration of the propagation pattern is shown in Figure 1.

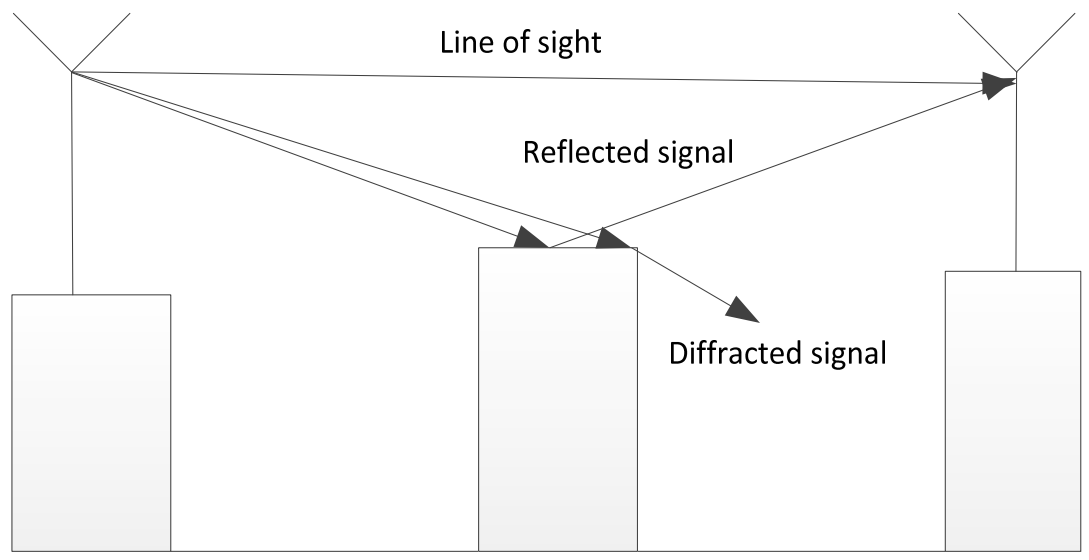

Fig. 1. Multipath propagation.

Thus, the resultant signal can be described as a superposition of a signal component propagating in different ways, and can be represented as:

$$
\begin{gathered}
S_{\Sigma}(t)=S_{l o s}(t)+S_{d i f}(t)+S_{r e f}(t)+S_{r d}(t)+ \\
+S_{d r}(t)+S_{r r}(t)+S_{d d}(t)
\end{gathered}
$$

The signal propagated within the line of sight makes the greatest contribution to the resulting signal. As has been shown in [7], the probability of occurrence of the signal within the line of sight can be described by the expression:

$$
P_{l}(0)=e^{-\gamma_{0} l}
$$

where $l$ - distance between the receiver and the transmitter,

$$
\gamma_{0}=\frac{2 v\langle L\rangle}{\pi},
$$

where $v$ - average density of screens, $\langle L\rangle$ - average screen length. 
Expression (4) is subject to a Poisson distribution.

Signals subjected to multiple diffraction have a large attenuation coefficient, so they do not contribute significantly to the resultant signal arriving at the receiver. Waves subjected to multiple diffraction are attenuated sufficiently strongly, so they have practically no effect on the resulting signal at the receiver [8].

In the case where the signal falls to the surface in a few times the wavelength occurs multipath signal.

The probability of signal reflections in a city described by the expression:

$$
P_{l}(m)=\frac{\left(\bar{m}_{l}\right)^{m}}{m !} e^{-\bar{m}_{l}}
$$

where $\bar{m}=\gamma_{0} l$ - the average number of intersections between the receiver and the transmitter.

This expression, as well as in the case of the likelihood of direct line of sight, obeys the Poisson distribution.

In general, a surface representing a walls of buildings and the ground surface, can be represented in the form of a screen with a rough surface, the height of unevenness of the surface of which depends on the coordinates, and varies according to the normal distribution law.

In the case when the height of the irregularities satisfies the conditions [9] $H \ll \frac{\lambda}{16 \sin (\psi)}$, then a mirror reflection of the wave from the surface occurs. If the conditions are not met, then there is half- scattered reflected wave. In scattering wave is reflected in accordance with the scattering phase function given by equation [10]

$$
\begin{aligned}
& J(\theta, \varphi)=\frac{S_{0}}{4 \pi D_{10}^{2} D_{20}^{2}}\left(\frac{h}{\sigma}\right)^{2}\left[\frac{1-\cos \psi \cos \theta \cos \varphi+\sin \psi \sin \theta}{\sin \psi+\sin \theta}\right]^{2} \times \\
& \times \exp \left(-\left(\frac{h}{2 \sigma}\right)^{2} \frac{\cos ^{2} \psi+\cos ^{2} \theta-2 \cos \psi \cos \theta \cos \varphi}{(\sin \psi+\sin \varphi)^{2}}\right)
\end{aligned}
$$

where $S_{0}$ - area of the reflecting surface; $D_{10}$ - distance from the source to the reflection surface; $D_{20}$ - the distance from the reflection surface to the receiver; $h$ - the height of the unevenness; $\sigma$ - dispersion of the height of the reflecting surf; $\psi-$ angle of slip; $\theta-$ elevation angle; $\varphi$ - azimuth.

The width of the cone with respect to the elevation and azimuth, provided that the wave falls onto the plane, is determined by the relations:

$$
\begin{gathered}
\Delta \theta \simeq \sqrt{8 \bar{\xi}_{x}^{2}}, \\
\Delta \varphi \simeq \sqrt{8 \operatorname{tg}^{2} \psi \bar{\xi}_{y}^{2}},
\end{gathered}
$$

where $\xi_{x}, \xi_{y}-$ the distribution of the inclination angles of the reflecting surface in the directions $x$ and $y$, respectively. 
In this case, the maximum energy of the signal is accounted for by the mirror component of the reflected wave. The remaining energy is scattered in accordance with the scattering indicatrix represented in expression (6).

To analyze the resulting signal, we consider the last section of the signal propagation along different paths. This signal consists of components $S_{l o s}(t), S_{d i f}(t), S_{r e f}(t)$, each of which can reach the receiver with a probability $P_{l}(0)$.

In this case, the components $S_{d i f}(t)$ and $S_{\text {ref }}(t)$ could reach the region under consideration by multiple reflections, diffraction, or combinations thereof. It should also be noted that the behavior of each of the reflected components depends on the location of the reflecting surface $S$, the angle of incidence $\psi$ of the wave on the reflecting surface.

Thus, the resulting signal arriving at the receiver antenna, in accordance with (2) and (3) may be represented by the following expression

$$
\begin{aligned}
S_{\Sigma}(t, \theta, \varphi)= & h_{l o s}(t, \tau, \theta, \varphi) s(t, P(0))+\sum_{l=1}^{L} h_{d i f}\left(t, \tau_{l}, \theta_{l}, \varphi_{l}\right)_{l} s(t, P(0))+ \\
& +\sum_{n=1}^{N} h_{r e f}\left(t, \theta_{n}, \varphi_{n}, \psi_{n}\right)_{n} s(t, P(0))+\eta
\end{aligned} .
$$

The impulse response of the signal components is determined as follows:

$$
\begin{gathered}
h(t, \tau)_{d i f}=a(t) G_{R X}(\theta, \varphi) e^{-j 2 \pi f_{c} \tau}, \\
h(t, \tau)_{d i f}=a_{d i f}(t) G_{R X}(\theta, \varphi) e^{-j 2 \pi f_{c} \tau}, \\
h(t, \tau)_{r e f}=R_{r e f} \sum_{\Delta \theta} \sum_{\Delta \varphi}\left[\begin{array}{l}
a(t) J(\Delta \theta, \Delta \varphi, \psi) \times \\
\times G_{R X}(\Delta \theta, \Delta \varphi) e^{-j 2 \pi f_{c} \tau}
\end{array}\right],
\end{gathered}
$$

where $a(t)$ - attenuation of signal, $G_{R X}(\theta, \varphi)$ - antenna gain, $f_{c}$ - carrier frequency, $R_{\text {ref }}$ - reflection coefficient of the reflecting surface.

In urban areas, increasing the distance between the receiver and the transmitter decreases the likelihood of line of sight (expression (4)), since the receiver installed on the mobile station moves along the streets and the gap between the houses appears briefly and infrequently. In this connection, it can be concluded that the direct propagation path will not make a significant contribution to the resulting signal from expression (9)

In an urban environment, increasing the distance between the receiver and the transmitter decreases the likelihood of line of sight (expression (4)), since the receiver installed on the mobile station moves along the streets and the gap between the houses appears briefly and infrequently. In this connection, it can be concluded that the line of sight propagation path will not make a significant contribution to the resulting signal from expression (10)

The simulation shows that the histogram of the signal (Figure 2) from expression (9) corresponds to the Rayleigh distribution. 


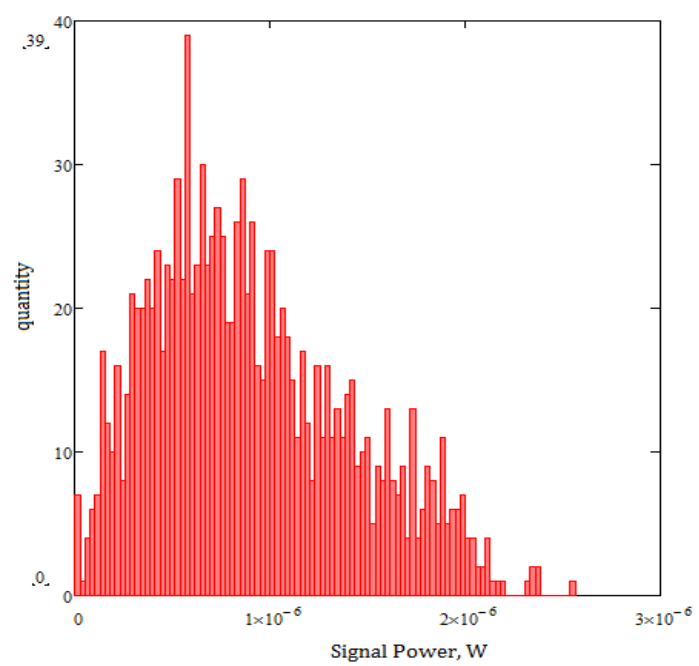

Fig. 2. The histogram of the signal.

\section{Conclusion}

This article was reviewed by the propagation mechanism in urban areas, and has been analyzed and the task of the development of three-dimensional model of the communication channel in the urban areas.

\section{References}

1. V. Fedosov, A. Legin, A. Lomakina, Serbian Journal of Electrical Engineering, 14(3), 301 (2017)

2. V. P. Fedosov, A.V. Emelyanenko, N.O. Ternovay, R.V. Rubtsov, V.I. Marchuk, 2014 12th International Conference on Signal Processing (ICSP), 1537 (2014) doi: 10.1109/ICOSP.2014.7015255

3. V.P. Fedosov, A.A. Legin, A.V. Lomakina, 2017 Radiation and Scattering of Electromagnetic Waves (RSEMW), 218 (2017) doi: 10.1109/RSEMW.2017.8103633

4. Spatial Channel Model for Multiple Input Multiple Output (MIMO) Simulation. 3GPP. Vol. TR 25.996, v6.1.0. (2003)

A. Kuchar, J.-P. Rossi, E. Bonek, IEEE Trans. Antennas Propagat., 48(2),137 (2000)

5. J. Laurila, K. Kalliola, M. Toeltsch, K. Hugl, P. Vainikainen, E. Bonek, IEEE Trans. Antennas Propagat., 50(2), 233 (2002)

6. Y. Karasawa, URSI Radio Science Bulletin, 2002(302), 5 (2002)

7. V.G. Gavrilenko, V.A. Yashnov, Propagation of radio waves in modern mobile communication systems (Lobachevsky State University of Nizhni Novgorod, Nizhny Novgorod, 2003).

8. F.B. Black, Propagation of radio waves (Sovetskoe radio, Moscow, 1972)

9. F.G. Bass, I.M. Fuchs, Scattering of waves on a statistically uneven surface (Ripol Classic-Nauka, Moscow, 1972)

10. A.A. Karpov, Journal of Radio Electronics, 8, 74 (2008)

11. Y.V. Lavrentyev, Journal of Radio Electronics, 5, 102 (2000) 